\title{
Algebraic Connections, Universal Bimodules and Entire Cyclic Cohomology
}

\author{
Masoud Khalkhali \\ Mathematisches Institut der Universität Heidelberg, Im Neuenheimer Feld 288, D-69120 Heidelberg, \\ Germany
}

Received: 15 June 1993

\begin{abstract}
A new method of constructing homotopies suitable for entire cyclic cohomology is presented. As a result, the periodic and entire cyclic cohomology of Banach algebras of finite cohomological dimension are shown to be isomorphic. The same method can be used to calculate the algebraic entire cyclic cohomology of (non-commutative) tori.
\end{abstract}

\section{Contents}

1. Introduction . . . . . . . . . . . . . . . . . . . . . . . . . 433

2. Definitions of Hochschild, Periodic Cyclic and Entire Cyclic

Cohomology . . . . . . . . . . . . . . . . . . . . . . . . . 434

3. Perturbation Lemma and Retraction Formulae . . . . . . . . . . . 436

4. Algebraic Connections, Universal Bimodules and Homotopy . . . . . 439

5. Proof of the Main Theorem . . . . . . . . . . . . . . . . . . 443

\section{Introduction}

In this paper we show that for a large class of Banach algebras $A$, the natural map $H P^{*}(A) \rightarrow H E^{*}(A)$, from the periodic cyclic cohomology of $A$ to the entire cyclic cohomology of $A$, is an isomorphism.

We prove this result assuming that there is an integer $n \geqq 0$ such that for all dual Banach $A$-bimodules $M^{*}$, the Hochschild cohomology $H^{n+1}\left(A, M^{*}\right)$ vanishes. Examples of such algebras are: amenable Banach algebras $(n=0$; see also [13] for an independent proof of this special case), and Banach algebras of finite Hochschild cohomological dimension. Note that by results of Connes [2] and Haagerup [7], a $C^{*}$-algebra is amenable if and only if it is nuclear.

Introduced by Alain Connes in [4], entire cyclic cohomology is an infinite dimensional version of cyclic cohomology and is an appropriate tool to handle index 
theory and analysis on infinite dimensional commutative and noncommutative spaces as well as supersymmetric quantum field theory. See [5,8 and 12] for motivating examples, applications and some further results in the theory. So, it is natural to expect that in finite dimensional situations entire cyclic cohomology would yield the same results as periodic cyclic cohomology.

The main idea in our proof is a new method of constructing contracting homotopies for the Hochschild complex with a good control on the size of the homotopies. As explained in [5], this is enough to show that the above natural map is an isomorphism. We use the theory of connections on bimodules and its cohomological interpretation due to Cuntz and Quillen [6] to construct the required homotopies. This theory generalizes the earlier work of Connes on connection and curvature in noncommutative setting. There is strong evidence that our results will also be useful to establish the isomorphism of periodic and entire cyclic cohomology of the algebra of smooth functions on a finite dimensional smooth manifold. This is currently under investigation.

The paper is organized as follows. In Sect. 2 we recall the definition and some of the basic properties of Hochschild, periodic cyclic and entire cyclic cohomology theories needed in this article. In Sect. 3 we derive some retraction formulae from [5] and prove a theorem from [14]. This result states that if there is a contracting homotopy of say uniformly bounded norm for the Hochschild complex of $A$, then the map $H P^{*}(A) \rightarrow H E^{*}(A)$ is an isomorphism. Our proof is based on homological perturbation theory and seems to be new. In Sect. 4, we recall the Cuntz-Quillen notion of a connection on an algebra [6]. We show that a connection can be used to construct the required homotopies. This is enough to prove the theorem for the case of finite cohomological dimension. As an application we calculate the (algebraic) entire cyclic cohomology of the circle and recover a theorem of Connes [5]. Finally in Sect. 5 we prove the main theorem of this article (Theorem 5.2). Here we assume less than finite cohomological dimension, so that connections do not exist anymore. However, we can still define good homotopies using cocycles with values in certain universal (double) dual bimodules.

I would like to express my deepest gratitude to Alain Connes and Joachim Cuntz for their encouragement, support and very interesting discussions on the subject of this article. I am also very grateful to Ersin Sümer for typing the manuscript.

\section{Definitions of Hochschild, Periodic Cyclic and Entire Cyclic Cohomology}

Here we recall basic definitions and some of the properties of Hochschild, periodic cyclic and entire cyclic cohomology theories [3, 5]. Let $A$ be an associative algebra over a field of characteristic zero. In this paper the ground field is $\mathbb{C}$. Let $M$ be an $A$-bimodule. For $n \geqq 0$ let $C^{n}(A, M)$ be the space of $n$-linear forms on $A$ with values in $M$. The Hochschild coboundary $\delta: C^{n}(A, M) \rightarrow C^{n+1}(A, M)$ is defined by

$$
\begin{aligned}
\delta f\left(a_{1}, \ldots, a_{n+1}\right)= & a_{1} f\left(a_{2}, \ldots, a_{n+1}\right) \\
& +\sum_{i=1}^{n}(-1)^{i} f\left(a_{1}, \ldots, a_{i} a_{i+1}, \ldots, a_{n+1}\right) \\
& +(-1)^{n+1} f\left(a_{1}, \ldots, a_{n}\right) a_{n+1} .
\end{aligned}
$$


The Hochschild Cohomology of $A$ with coefficients in $M$, denoted by $H^{*}(A, M)$, is the cohomology of the complex

$$
0 \longrightarrow C^{0}(A, M) \stackrel{\delta}{\longrightarrow} C^{1}(A, M) \stackrel{\delta}{\longrightarrow} \cdots .
$$

We say $A$ has Hochschild cohomological dimension $n \geqq 0$, if for all bimodules $M, H^{n+1}(A, M)=0$ and there is a bimodule $N$ such that $H^{n}(A, N) \neq 0$.

Let $M^{*}$ be the space of linear functionals on $M$. It is naturally an $A$-bimodule via

$$
(a \phi b)(m)=\phi(b m a) \quad \forall a, b \in A, \phi \in M^{*} \text { and } m \in M .
$$

Such bimodules are called dual bimodules. In particular $A^{*}$ is a dual bimodule over $A$.

Let $C^{n}=C^{n}(A)=C^{n}\left(A, A^{*}\right)$. This can be identified as the space of $n+1$-linear functionals on $A$, where an $n$-cochain $f$ on $A$ with values in $A^{*}$ is identified with an $n+1$-linear functional $\phi$ on $A$, defined by $\phi\left(a_{0}, \ldots, a_{n}\right)=f\left(a_{1}, \ldots, a_{n}\right)\left(a_{0}\right)$. Under this identification, the Hochschild coboundary is identified with the operator $b: C^{n} \rightarrow$ $C^{n+1}$ defined by

$$
\begin{aligned}
b \phi\left(a_{0}, \ldots, a_{n+1}\right)= & \sum_{i=0}^{n}(-1)^{i} \phi\left(a_{0}, \ldots, a_{i} a_{i+1}, \ldots, a_{n+1}\right) \\
& +(-1)^{n+1} \phi\left(a_{n+1} a_{0}, a_{1}, \ldots, a_{n}\right) .
\end{aligned}
$$

Connes' boundary operator $B: C^{n+1} \rightarrow C^{n}$ is defined via

$$
\begin{aligned}
B \phi\left(a_{0}, \ldots, a_{n}\right)= & \sum_{i=0}^{n}(-1)^{n l}\left(\phi\left(1, a_{i}, \ldots, a_{n}, a_{0} \ldots, a_{l-1}\right)\right. \\
& \left.+(-1)^{n} \phi\left(a_{i}, \ldots, a_{n}, a_{0}, \ldots, a_{i-1}, 1\right)\right) .
\end{aligned}
$$

Note that for this to make sense, the algebra $A$ must be unital. We have $b^{2}=0$, $B^{2}=0$ and $b B+B b=0$.

Both theories, the periodic cyclic and entire cyclic cohomology, are defined via the Connes $(b, B)$ bicomplex $\mathcal{B}(A)=\left(C^{n, m}(A), b, B\right)$, where $C^{n, m}(A)=$ $C^{n-m}(A) ; n, m \in \mathbb{Z}$. The total complex of $\mathcal{B}(A)$ is a $\mathbb{Z} / 2$-graded complex

$$
C^{\mathrm{ev}} \underset{b+B}{\stackrel{b+B}{\rightleftarrows}} C^{\mathrm{odd}}
$$

where $C^{\mathrm{ev}}=\bigoplus_{n \geq 0} C^{2 n}$ and $C^{\text {odd }}=\bigoplus_{n \geq 0} C^{2 n+1}$. The periodic cyclic cohomology of $A$, denoted by $H P^{*}(A)$, is defined to be the cohomology of the above periodic complex.

In [4], Connes has observed that the complex of cochains with infinite support

$$
\prod_{n \geqq 0} C^{2 n} \underset{b+B}{\stackrel{b+B}{\rightleftarrows}} \prod_{n \geqq 0} C^{2 n+1}
$$

has always trivial homology, but that this need not be so if one restricts to infinite cochains $\left(\phi_{n}\right)_{n} \geqq 0$, in which the "size" of $\phi_{n}$ is suitably controlled. In order for this to be meaningful we restrict to locally convex algebras and continuous cochains. 
Definition 2.1. (cf. [5]). Let $A$ be a locally convex algebra. $A$ cochain $\left(\phi_{2 n}\right)$ (resp. $\left.\left(\phi_{2 n+1}\right)\right)$ is called entire if for any bounded subset $\sum$ of $A$ and $\lambda>0$ there exists $C=C_{\Sigma, \lambda}$ such that $\forall n \geqq 0$,

$$
\left|\phi_{2 n}\left(a_{0}, \ldots, a_{2 n}\right)\right| \leqq C \frac{\lambda^{n}}{n !} \quad \forall a_{i} \in \Sigma,
$$

and similarly in the odd case.

Let $C_{\varepsilon}^{\mathrm{ev}}$ (resp. $C_{\varepsilon}^{\mathrm{odd}}$ ) be the space of even (resp. odd) entire cochains on A. The cohomology of the $\mathbb{Z} / 2$-graded complex

$$
C_{\varepsilon}^{\mathrm{ev}} \underset{b+B}{\stackrel{b+B}{\rightleftarrows}} C_{\varepsilon}^{\mathrm{odd}}
$$

is by definition, the entire cyclic cohomology of the locally convex algebra $A$ and is denoted by $H E^{*}(A)$.

It is clear that a cochain of finite support is entire in the above sense, and hence we have a natural chain map $\left(C^{*}, b+B\right) \rightarrow\left(C_{\varepsilon}^{*}, b+B\right)$ which induces a map

$$
H P^{*}(A) \longrightarrow H E^{*}(A)
$$

from periodic to entire cyclic cohomology.

The two categories of locally convex algebras that are of interest in this paper are Banach algebras and arbitrary algebras endowed with the finest locally convex topology. It is not difficult to check that for a Banach algebra the above entire growth condition is equivalent to the radius of convergence of the power series

$$
\sum_{n \geqq 0}\left\|\phi_{2 n}\right\| \frac{(2 n) !}{n !} z^{n}
$$

being infinity [4], and hence the name entire cyclic cohomology. Here the norm of multilinear functionals is induced from the norm on $A$ via

$$
\left\|\phi_{n}\right\|=\sup \left\{\left|\phi_{n}\left(a_{0}, \ldots, a_{n}\right)\right| ;\left\|a_{i}\right\| \leqq 1\right\} .
$$

On the other extreme, as explained in [5], any algebra $A$ over $\mathbb{C}$ can be equipped with the finest locally convex topology (the weakest topology that all semi-norms are continuous), and is a locally convex algebra. The bounded subsets of $A$ are convex hulls of finite subsets $\Sigma$ of $A$ [5]. Thus a cochain $\phi=\left(\phi_{2 n}\right)$ is entire if for any finite subset $\Sigma$ of $A$ and $\lambda>0$ there exists $C=C_{\Sigma, \lambda}$ such that for all $n \geqq 0$,

$$
\left|\phi_{2 n}\left(a_{0}, \ldots, a_{2 n}\right)\right| \leqq C \frac{\lambda^{n}}{n !} \quad \forall a_{i} \in \Sigma
$$

\section{Perturbation Lemma and Retraction Formulae}

In this section we give a new derivation of some, more or less standard, retraction formulae from [5]. Our proof is based on homological perturbation theory [1], advocated by Kassel [11] for its applications to cyclic cohomology. In fact a simple version of the perturbation lemma of [1] and [11], formulated and proved below, is enough for our purpose. 
In the following we work with $\mathbb{Z} / 2$-graded complexes. Recall that a complex $(L, b)$ is a special deformation retract of a complex $(M, b)$ if there are chain maps

$$
L \stackrel{i}{\longrightarrow} M \stackrel{r}{\longrightarrow} L
$$

and a homotopy $h: M \rightarrow M$, of odd degree, such that

$$
\begin{aligned}
& r i=1_{L}, \\
& i r=1_{M}+b h+h b, \\
& h i=0 .
\end{aligned}
$$

Let us perturb the differentials to $b+B$ and assume $B i=i B$. It is natural to ask whether $(L, b+B)$ remains a deformation retract of $(M, b+B)$. The following simple version of the perturbation lemma of [1] and [11] shows that this is indeed the case, provided the infinite sum which defines the operator $K$ below can be rigorously defined. In our two applications of this lemma, this is guaranteed either by the presence of a filteration or by growth conditions.

Let $K: M \rightarrow M$ be the operator

$$
K=\sum_{n=0}^{\infty}(B h)^{n} .
$$

Define maps

$$
L \stackrel{I}{\longrightarrow} M \stackrel{R}{\longrightarrow} L
$$

and a homotopy $H: M \rightarrow M$ by $R=r K, H=h K$ and $I=i$.

Lemma 3.1 (perturbation lemma). The complex $(L, b+B)$ is a deformation retract of $(M, b+B)$.

Proof. The following two relations, valid for $n \geqq 1$, can be proved by induction:

$$
\left[r(B h)^{n}, b\right]=-\left[r(B h)^{n-1}, B\right],
$$

and

$$
h\left[(B h)^{n}, b\right]=-h(B h)^{n-1} B .
$$

Adding up (1) for $n \geqq 1$, we obtain

$$
[r K, b]=-[r K, B]
$$

or

$$
[R, b+B]=0,
$$

which shows $R$ is a morphism of $b+B$ complexes.

Similarly, adding up (2) for $n \geqq 1$, we obtain

$$
h[K, b]=-h K B,
$$

from which the homotopy formula

$$
I R=1_{M}+(b+B) H+H(b+B)
$$

easily follows. The rest is obvious.

Now, assume there are contracting homotopies 


$$
\alpha: C^{n}(A) \longrightarrow C^{n-1}(A) \quad n \geqq 2 N+1
$$

such that

$$
b \alpha+\alpha b=\mathrm{id} .
$$

Define a truncated $\mathbb{Z} / 2$-graded complex $C_{0}^{*}$ by

$$
C_{0}^{\mathrm{ev}}=\bigoplus_{n<N} C^{2 n} \oplus \operatorname{ker}\left\{b: C^{2 N} \rightarrow C^{2 N+1}\right\}
$$

and

$$
C_{0}^{\text {odd }}=\bigoplus_{n \leqq N} C^{2 n-1}
$$

Let us show that $\left(C_{0}^{*}, b\right)$ is a deformation retract of both $\left(C^{*}, b\right)$ and $\left(C_{\varepsilon}^{*}, b\right)$. Thus, let $i: C_{0}^{*} \rightarrow C^{*}$ be the canonical inclusion and define $r: C^{*} \rightarrow C_{0}^{*}$ by

$$
r \phi=\left(\phi_{0}, \ldots, \phi_{2 n-2}, \phi_{2 n}-\alpha b \phi_{2 n}\right)
$$

and

$$
r \phi=\left(\phi_{1}, \phi_{3}, \ldots, \phi_{2 n-1}\right)
$$

on even and odd cochains, respectively. Also, let the homotopy $h: C^{*} \rightarrow C^{*+1}$ be the diagonal operator defined by

$$
\begin{array}{ll}
h=-\alpha & n \geqq 2 N+1 \\
h=\quad 0 & n<2 N+1 .
\end{array}
$$

One easily checks that

$$
\begin{aligned}
& r i=1, \\
& i r=1+b h+h b,
\end{aligned}
$$

and

$$
h i=0 \text {. }
$$

Thus $\left(C_{0}^{*}, b\right)$ is a special deformation retract of $\left(C^{*}, b\right)$. The same argument shows that $\left(C_{0}^{*}, b\right)$ is also a special deformation retract of $\left(C_{\varepsilon}^{*}, b\right)$, provided $\|\alpha\|$ is of polynominal growth.

Now, by perturbation lemma $\left(C_{0}^{*}, b+B\right)$ is a deformation retract of $\left(C^{*}, b+B\right)$. Note that there is no convergence problem here. Indeed the retraction

$$
R: C^{*} \longrightarrow C_{0}^{*}
$$

and homotopy

$$
H: C^{*} \longrightarrow C^{*+1}
$$

on an even cochain $\phi=\left(\phi_{2 n}\right)$ are given by

$$
R \phi=r K \phi=\left((K \phi)_{0}, \ldots,(K \phi)_{2 N-2},(1-\alpha b)(K \phi)_{2 N}\right)
$$

and

$$
H \phi=h K \phi=\left(0, \ldots, 0,-\alpha(K \phi)_{2 N+2},-\alpha(K \phi)_{2 N+4}, \ldots\right) .
$$

Here the operator

$$
K: C^{*} \longrightarrow C^{*}
$$


is of the form

$$
\begin{aligned}
(K \phi)_{2 n} & =\sum_{j=0}^{\infty}(B h)^{j} \phi_{2 n+2 j} \\
& =\sum_{j=N-n+1}^{\infty}(-1)^{j}(B \alpha)^{j} \phi_{2 n+2 j} .
\end{aligned}
$$

Note that the infinite sums are actually finite and all the operators are welldefined.

Moreover, as is observed in [14], the same formula works in the entire case provided $\|\alpha\|$ is uniformly bounded. The proof is straightforward and we omit it. We have thus shown that $\left(C_{0}^{*}, b+B\right)$ is a deformation retract of $\left(C_{\varepsilon}^{*}, b+B\right)$, provided $\|\alpha\|$ is uniformly bounded.

Now consider the diagram of chain maps

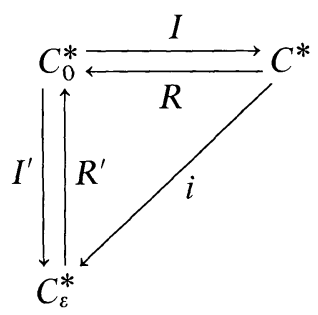

We have $R^{\prime} i=R$, where $i$ is the natural inclusion. Since $R^{*}=R^{*}=\mathrm{id}$, we get $i^{*}=\mathrm{id}$. We have thus proved the following theorem from [14].

Theorem 3.2. The natural inclusion $\left(C^{*}, b+B\right) \rightarrow\left(C_{\varepsilon}^{*}, b+B\right)$ is a quasiisomorphism, provided $\|\alpha\|$ is uniformly bounded.

\section{Algebraic Connections, Universal Bimodules and Homotopy}

In this section we first recall the theory of connections on noncommutative differential forms and its cohomological interpretation due to Cuntz and Quillen [6]. We then show how one can construct homotopies with good properties, using a connection or its defining cochain. As an application we calculate the (algebraic) entire cyclic cohomology of the circle and recover a theorem of Connes [5]. In the next section we apply our formalism to prove the main theorem of this paper.

Let $A$ be a unital algebra over $\mathbb{C}$ and let $\Omega A=\oplus_{n} \geqq 0 \Omega^{n} A$ be the differential graded algebra of noncommutative differential forms over $A$. One can think of $\Omega A$ as the universal unital $D G$ algebra generated by $A$ in degree zero. See $[3,6,10]$ for details.

Let $\bar{A}=A / \mathbb{C}$. The normalized Hochschild complex $\left(A \otimes A^{-\otimes n}, b\right)$ which calculates Hochschild homology $H_{*}(A, A)$ can be identified with $\Omega A$ via

$$
a_{0} d a_{1} \ldots d a_{n} \simeq\left(a_{0}, a_{1}, \ldots, a_{n}\right),
$$

where $\left(a_{0}, a_{1}, \ldots, a_{n}\right)$ denotes the class of $a_{0} \otimes a_{1} \otimes \cdots \otimes a_{n}$ [6]. Under this identification, the Hochschild boundary $b$ is transformed to differential forms and is 
given by [6]

$$
b(\omega d a)=(-1)^{|\omega|}[\omega, a]=(-1)^{|\omega|}(\omega a-a \omega) .
$$

Let $n \geqq 0$. A (right) $n$-connection on $A$, in the sense of Cuntz-Quillen [6], is a linear map

$$
\nabla: \Omega^{n} A \longrightarrow \Omega^{n+1} A,
$$

such that $\forall a \in A$ and $\forall \omega \in \Omega^{n} A$,

$$
\nabla a \omega=a \nabla \omega
$$

and

$$
\nabla \omega a=\nabla \omega \cdot a+\omega d a .
$$

Given an $n$-connection $\nabla$ on $A$, define as in [6] an $n$-cochain $\phi$ on $A$ with values in the $A$-bimodule $\Omega^{n+1} A$,

$$
\phi: A^{\otimes n} \rightarrow \Omega^{n+1} A,
$$

by

$$
\phi\left(a_{1}, \ldots, a_{n}\right)=\nabla\left(d a_{1} \ldots d a_{n}\right) .
$$

Using (4.2), it is not difficult to check that

$$
\delta \phi\left(a_{1}, \ldots, a_{n+1}\right)=(-1)^{n} d a_{1} \ldots d a_{n+1} .
$$

Conversely, given a cochain $\phi$ satisfying (6), we can define a connection $\nabla$ by putting

$$
\nabla\left(a_{0} d a_{1} \ldots d a_{n}\right)=a_{0} \phi\left(a_{1}, \ldots, a_{n}\right) .
$$

Let us show as in [6] that an $n$-connection on $A$ exists, if and only if $H^{n+1}(A, M)=0$ for all $A$-bimodules $M$; i.e. Hochschild cohomological dimension of $A$ is at most $n$. To see this, define a cochain

$$
d^{n+1}: A^{\otimes n+1} \longrightarrow \Omega^{n+1} A
$$

by

$$
d^{n+1}\left(a_{1}, \ldots, a_{n+1}\right)=d a_{1} \ldots d a_{n+1} .
$$

It is easy to check that $d^{n+1}$ is a cocycle and is indeed a universal cocycle in the sense that any normalized cocycle $\psi: A^{\otimes n+1} \rightarrow M$ is uniquely induced by a bimodule map $\Pi: \Omega^{n+1} A \rightarrow M$ (define $\Pi$ by $\Pi\left(a_{0} d a_{1} \ldots d a_{n+1}\right)=a_{0} \psi\left(a_{1}, \ldots, a_{n+1}\right)$ ).

Thus, by functoriality, $H^{n+1}(A, M)=0$ for all $M$ if and only if $d^{n+1}$ is a coboundary. That is, if a cochain $\phi: A^{\otimes n+1} \longrightarrow \Omega^{n+1} A$ exists that satisfy (6). This is of course equivalent to the existence of an $n$-connection on $A$.

Now, let us show that connections can be used to construct contracting homotopies for the Hochschild complex beyond the cohomological dimension.

Let $\nabla$ be an $n$-connection on $A$. Define

$$
\alpha: \Omega^{k} A \longrightarrow \Omega^{k+1} A \quad k \geqq n,
$$

by

$$
\alpha\left(a_{0} d a_{1} \ldots d a_{k}\right)=\nabla\left(a_{0} d a_{1} \ldots d a_{n}\right) d a_{n+1} \ldots d a_{k} .
$$

Lemma 4.1. For all $\omega \in \Omega^{k} A$ and $a \in A$, we have $\alpha(a \omega)=a \alpha(\omega)$ and $\alpha(\omega a)$ $=\alpha(\omega) a+(-1)^{|\omega|-n} \omega d a$. 
Proof. The first relation is obvious. To prove the second relation let $\omega=$ $a_{0} d a_{1} \ldots d a_{k}$. We have

$$
\begin{aligned}
\omega a= & a_{0} d a_{1} \ldots d a_{k} \cdot a=a_{0} d a_{1} \ldots d\left(a_{k} a\right) \\
& +\sum_{l=1}^{k-1}(-1)^{i+k} a_{0} d a_{1} \ldots d\left(a_{i} a_{i+1}\right) \ldots d a_{k} d a \\
& +(-1)^{k} a_{0} a_{1} d a_{2} \ldots d a_{k} d a
\end{aligned}
$$

Thus,

$$
\begin{aligned}
\alpha(\omega a)= & \nabla\left(a_{0} d a_{1} \ldots d a_{n}\right)\left\{d a_{n+1} \ldots d\left(a_{k} a\right)\right. \\
& \left.+\sum_{i=n+1}^{k-1}(-1)^{i+k} d a_{n+1} \ldots d\left(a_{i} a_{i+1}\right) \ldots d a_{k} d a\right\} \\
& +\nabla\left\{\sum_{i=1}^{n}(-1)^{i+k} a_{0} d a_{1} \ldots d\left(a_{i} a_{i+1}\right) \ldots d a_{n+1}\right. \\
& \left.+(-1)^{k} a_{0} a_{1} d a_{2} \ldots d a_{n+1}\right\} \cdot d a_{n+2} \ldots d a_{k} d a \\
= & \nabla\left(a_{0} d a_{1} \ldots d a_{n}\right)\left\{d a_{n+1} \ldots d a_{k} \cdot a+(-1)^{n+k+1} a_{n+1} d a_{n+2} \ldots d a_{k} d a\right\} \\
& +(-1)^{n+k} \nabla\left(a_{0} d a_{1} \ldots d a_{n} \cdot a_{n+1}\right) d a_{n+2} \ldots d a_{k} d a .
\end{aligned}
$$

Using the right connection property, this is equal to

$$
\begin{aligned}
= & \nabla\left(a_{0} d a_{1} \ldots d a_{n}\right) d a_{n+1} \ldots d a_{k} \cdot a \\
& +(-1)^{n+k+1} \nabla\left(a_{0} d a_{1} \ldots d a_{n}\right) a_{n+1} d a_{n+2} \ldots d a_{k} d a \\
& +(-1)^{n+k} \nabla\left(a_{0} d a_{1} \ldots d a_{n}\right) a_{n+1} d a_{n+2} \ldots d a_{k} d a \\
& +(-1)^{n+k} a_{0} d a_{1} \ldots d a_{n} d a_{n+1} \ldots d a_{k} d a \\
= & \alpha(\omega) a+(-1)^{|\omega|-n} \omega d a .
\end{aligned}
$$

As a corollary, we can now prove

Proposition 4.2. In dimensions $\geqq n+1$, we have $b \alpha+\alpha b=(-1)^{n}$ id.

Proof. Choose a differential form $\omega d a$ of degree $\geqq n+1$. We have $\alpha(\omega d a)=$ $\alpha(\omega) d a$. Thus, using formula (5) for $b$, we get

$$
b \alpha(\omega d a)=(-1)^{|\omega|+1}[\alpha(\omega), a]=(-1)^{|\omega|+1}(\alpha(\omega) a-a \alpha(\omega)) .
$$

Also,

$$
\begin{aligned}
\alpha b(\omega d a) & =(-1)^{|\omega|} \alpha(\omega a-a \omega) \\
& =(-1)^{|\omega|}\left(\alpha(\omega) a+(-1)^{|\omega|-n} \omega d a-a \alpha(\omega)\right) .
\end{aligned}
$$

So that

$$
(b \alpha+\alpha b)(\omega d a)=(-1)^{n} \omega d a,
$$

or

$$
b \alpha+\alpha b=(-1)^{n} \mathrm{id} .
$$


Example. (Algebraic) entire cyclic cohomology of the circle. Let $A=\mathbb{C}\left[x, x^{-1}\right]$ be the algebra of Laurent polynomials (algebraic functions on $S^{1}$ ). This algebra has Hochschild cohomological dimension 1 . Indeed it is easy to see that $\Omega^{1} A$ is a free $A$-bimodule on a single generator $d x$, and thus the space of 1-connections on $A$

$$
\nabla: \Omega^{1} A \longrightarrow \Omega^{2} A
$$

is an affine space over the vector space $\Omega^{2} A$ : any choice of $\nabla(d x) \in \Omega^{2} A$ uniquely defines a connection via

$$
\nabla(f d x \cdot g)=f \nabla(d x) g+f d x d g \quad \forall f, g \in A .
$$

Let us choose $\nabla(d x)=0$. Then we have

$$
\nabla\left(d x^{n}\right)=\sum_{i=0}^{n-2} x^{n-2-i} d x d x^{i+1} \quad n \geqq 2
$$

and

$$
\nabla\left(d x^{-n}\right)=-\sum_{i=0}^{n-1} x^{-n+i} d x d x^{-i-1} \quad n \geqq 1
$$

The formulae obtained for the homotopy $\alpha: \Omega^{k} A \rightarrow \Omega^{k+1} A, k \geqq 1$, using (7), is close to but different from the homotopy given by Connes in his calculation of the algebraic entire cyclic cohomology of $S^{1}$ [5, Sect. IV.6.]. Let us check that the perturbation series of Sect. 2 acutally converge and thus show that $H P^{*}(A) \simeq$ $H E^{*}(A)$.

Let $\phi=\left(\phi_{2 n}\right)_{n} \geqq 0$ be an even entire cocylce on $A$. From the homotopy formula $I R=1+(b+B) H+H(b+B)$ of Sect. 2 , we have

$$
\phi-\tilde{\phi}=(b+B) \psi,
$$

where

$$
\tilde{\phi}=\left(\tilde{\phi}_{0}, 0,0, \ldots\right), \quad \tilde{\phi}_{0}=\sum_{j=0}^{\infty}(-1)^{j}(B \alpha)^{j} \phi_{2 j}
$$

and

$$
\psi=\left(\psi_{2 n+1}\right), \psi_{2 n+1}=\sum_{j=0}^{\infty}(-1)^{j} \alpha(B \alpha)^{j} \psi_{2 n+2 j+2} .
$$

We have to show the series are (pointwise) convergent and $\psi$ is an entire cochain.

To see this, for each $p \geqq 1$ define a finite set

$$
\Sigma=\left\{x^{-p}, x^{-p+1}, \ldots, 1, x, \ldots, x^{p}\right\} .
$$

Lemma 4.3. Let $a_{i} \in \Sigma, 0 \leqq i \leqq n$. Then $\alpha B\left(a_{0} d a_{1} \ldots d a_{n}\right)$ is a linear combination of at most $p(n+1)$ terms of the form $b_{0} d b_{1} \ldots d b_{n+2}$ where $b_{i} \in \Sigma$.

Proof. Note that $B\left(a_{0} d a_{1} \ldots d a_{n}\right)$ is a linear combination of at most $(n+1)$ terms of the form $d c_{1} d c_{2} \ldots d c_{n+1}, c_{i} \in \Sigma$. Now, we have

$$
\alpha\left(d c_{1} d c_{2} \ldots d c_{n+1}\right)=\nabla\left(d c_{1}\right) d c_{2} \ldots d c_{n+1} .
$$

The rest follows from formulae (8) for $\nabla\left(d x^{n}\right)$ and $\nabla\left(d x^{-n}\right)$. 
Now we can prove the pointwise convergence of the above series. By entire growth condition on $\phi$ and the above lemma, we have for $a \in \Sigma$,

$$
\begin{aligned}
\left|(B \alpha)^{j} \phi_{2 j}(a)\right| & =\left|\phi_{2 j}(\alpha B \alpha B \ldots \alpha B(a))\right| \\
& \leqq C \lambda^{j} p^{j}(2 j-1)(2 j-3) \ldots 5 \cdot 3 \cdot 1 \cdot \frac{1}{j !} \\
& \leqq C(2 p \lambda)^{j}
\end{aligned}
$$

where $C=C_{\Sigma, \lambda}$. Choosing $\lambda$ such that $2 p \lambda<1$, we see that the first series converges. To check the convergence and entire growth condition for $\psi=\left(\psi_{2 n+1}\right)$ note that, by linearity, it suffices to work with finite sets of the above type. We then have

$$
\begin{aligned}
& \left|\psi_{2 n+1}\left(a_{0} d a_{1} \ldots d a_{2 n+1}\right)\right| \\
& \quad \leqq \sum_{j=0}^{\infty}\left|\alpha(B \alpha)^{j} \phi_{2 n+2 j+2}\left(a_{0} d a_{1} \ldots d a_{2 n+1}\right)\right| \\
& \quad \leqq \sum_{j=0}^{\infty} C \lambda^{n+j+1}(2 n+2 j+1)(2 n+2 j-1) \ldots(2 n+3) p^{j} \frac{1}{(n+j+1) !} \\
& \quad \leqq \frac{C \lambda^{n+1}}{n !} \sum_{j=0}^{\infty}(2 p \lambda)^{j} .
\end{aligned}
$$

The estimate both implies the convergence and entire growth condition for $\psi=\left(\psi_{2 n+1}\right)$.

We have thus obtained a proof of the following result of Connes [5, Sect. IV.6, Theorem 25]

Theorem 4.4. The entire cyclic cohomology of the algebra of Laurent polynomials $A=\mathbb{C}\left[x, x^{-1}\right]$ is given by

$$
H E^{\mathrm{ev}}(A) \simeq \mathbb{C}, H E^{\mathrm{odd}}(A) \simeq \mathbb{C}
$$

The generators are given by the cyclic cocycles

$$
\tau_{0}\left(f=\int f(x) d x, \tau_{1}\left(f^{0}, f^{1}\right)=\int f^{0} d f^{1}\right.
$$

With some more work and using the same approach, one can prove similar results for higher dimensional (noncommutative) tori.

\section{Proof of the Main Theorem}

As a second application of our approach, in this section we prove the main theorem of this article (Theorem 5.2 below). Thus, let $A$ be a Banach algebra and assume there is a fixed integer $n \geqq 0$ such that for all dual Banach $A$-bimodules $M^{*}$, the Hochschild cohomology $H^{n+1}\left(A, M^{*}\right)$ vanishes. See [9] for elements of Hochschild cohomology for Banach algebras. Let us show how to construct "good" contracting homotopies for the Hochschild complex $\left(C^{*}(A), b\right)$. 
Let us endow each $\Omega^{k} A, k \geqq 0$, with the projective tensor product norm and use the same notation to denote the completion. Thus, in this section, $\Omega^{k} A \simeq A \hat{\otimes} A^{-\hat{\otimes} k}$. Let

$$
d^{n+1}: A^{\otimes(n+1)} \longrightarrow\left(\Omega^{n+1} A\right)^{* *}
$$

be the cocycle defined by

$$
d^{n+1}\left(a_{1}, \ldots, a_{n+1}\right)=d a_{1} \ldots d a_{n+1},
$$

where we have implicitly used the canonical embedding

$$
\Omega^{n+1} A \longrightarrow\left(\Omega^{n+1} A\right)^{* *} \text {. }
$$

By our assumption, $H^{n+1}\left(A,\left(\Omega^{n+1} A\right)^{* *}\right)=0$. Thus, there is a cochain

$$
\phi: A^{\otimes n} \longrightarrow\left(\Omega^{n+1} A\right)^{* *}
$$

such that

Now, define

$$
\delta \phi=d^{n+1} .
$$

$$
\alpha:\left(\Omega^{k+1} A\right)^{*} \longrightarrow\left(\Omega^{k} A\right)^{*} \quad k \geqq n
$$

via

$$
(\alpha f)\left(a_{0} d a_{1} \ldots d a_{k}\right)=a_{0} \phi\left(a_{1}, \ldots, a_{n}\right)\left(f_{d a_{n+1} \ldots d a_{k}}\right),
$$

where for $f \in\left(\Omega^{k+1} A\right)^{*}, f_{d a_{n+1} \ldots d a_{k}} \in\left(\Omega^{n+1} A\right)^{*}$ is defined by

$$
f_{d a_{n+1} \ldots d a_{k}}(\omega)=f\left(\omega d a_{n+1} \ldots d a_{k}\right) \quad \forall \omega \in \Omega^{n+1} A .
$$

Note that the complex $\left(\left(\Omega^{k} A\right)^{*}, b\right)$ is canonically isomorphic to the normalized Hochschild complex $\left(C^{k}(A), b\right)_{\text {norm }}$ which calculates the Hochschild cohomology. We could similarly construct contracting homotopies for $\left(C^{k}(A), b\right)$, by simply working with the nonunital version of $\Omega A$ and identifying $\left(C^{k}(A), b\right)$ with a subcomplex of $\left(\left(\Omega^{k} A\right)^{*}, b\right)$. Thus we can avoid using simplicially normalized entire $(b, B)$-bicomplex if we choose to.

Proposition 5.1. In dimensions $\geqq n+1$, we have $b \alpha+\alpha b=$ id.

Proof. Let $k \geqq n$. We have, for $f \in\left(\Omega^{k+1} A\right)^{*}$,

$$
\begin{aligned}
b(\alpha f) & \left(a_{0} d a_{1} \ldots d a_{k+1}\right) \\
= & (-1)^{k}(\alpha f)\left(a_{0} d a_{1} \ldots d a_{k} \cdot a_{k+1}-a_{k+1} a_{0} d a_{1} \ldots d a_{k}\right) \\
= & (-1)^{k}(\alpha f)\left\{\sum_{i=n+1}^{k}(-1)^{i+k} a_{0} d a_{1} \ldots d a_{n} d a_{n+1} \ldots d a_{i} a_{i+1} \ldots d a_{k+1}\right. \\
& +\sum_{i=1}^{n}(-1)^{i+k} a_{0} d a_{1} \ldots d a_{i} a_{i+1} \ldots d a_{n+1} d a_{n+2} \ldots d a_{k+1} \\
& +(-1)^{k} a_{0} a_{1} d a_{2} \ldots d a_{n+1} d a_{n+2} \ldots d a_{k+1} \\
& \left.-a_{k+1} a_{0} d a_{1} \ldots d a_{k}\right\} \\
= & (-1)^{k} a_{0} \phi\left(a_{1}, \ldots, a_{n}\right)\left(f_{\omega_{0}}\right)+a_{0}\left(a_{1} \phi\left(a_{2}, \ldots, a_{n+1}\right)\right. \\
& \left.+\sum_{i=1}^{n}(-1)^{i} \phi\left(a_{1}, \ldots, a_{i} a_{i+1}, \ldots, a_{n+1}\right)\right)\left(f_{\left.d a_{n+2} \ldots d a_{k+1}\right)}\right) \\
& -(-1)^{k} a_{k+1} a_{0} \phi\left(a_{1}, \ldots, a_{n}\right)\left(f_{d a_{n+1} \ldots d a_{k}}\right),
\end{aligned}
$$


where $\omega_{0}=\sum_{i=n+1}^{k}(-1)^{i+k} a_{0} d a_{1} \ldots d a_{n} d a_{n+1} \ldots d a_{i} a_{i+1} \ldots d a_{k+1}$.

Using $\delta \phi=d^{n+1}$, this can be written as

$$
\begin{aligned}
= & (-1)^{k} a_{0} \phi\left(a_{1}, \ldots, a_{n}\right)\left(f_{\omega_{0}}\right)+a_{0}\left\{d a_{1} \ldots d a_{n+1}+(-1)^{n} \phi\left(a_{1}, \ldots, a_{n}\right) a_{n+1}\right. \\
& \left.\left(f_{d a_{n+2} \ldots d a_{k+1}}\right)\right\}-(-1)^{k} a_{k+1} a_{0} \phi\left(a_{1}, \ldots, a_{n}\right)\left(f_{d a_{n+1} \ldots d a_{k}}\right) .
\end{aligned}
$$

Note that $a_{0} d a_{1} \ldots d a_{n+1}\left(f_{d a_{n+2} \ldots d a_{k+1}}\right)=f\left(a_{0} d a_{1} \ldots d a_{k+1}\right)$. On the other hand we have

$$
\alpha(b f)\left(a_{0} d a_{1} \ldots d a_{k+1}\right)=a_{0} \phi\left(a_{1}, \ldots, a_{n}\right)\left((b f)_{d a_{n+1} \ldots d a_{k+1}}\right) .
$$

Now, for all $\omega$ in $\Omega^{n+1} A$,

$$
\begin{aligned}
(b f)_{d a_{n+1} \ldots d a_{k+1}}(\omega)= & b f\left(\omega d a_{n+1} \ldots d a_{k+1}\right) \\
= & (-1)^{k+1} f\left(\omega d a_{n+1} \ldots d a_{k} \cdot a_{k+1}-a_{k+1} \omega d a_{n+1} \ldots d a_{k}\right) \\
= & (-1)^{k+1} f\left(\omega \alpha+(-1)^{n} \omega a_{n+1} d a_{n+2} \ldots d a_{k+1}\right) \\
& -(-1)^{k} f\left(a_{k+1} \omega d a_{n+1} \ldots d a_{k}\right) \\
= & \left\{(-1)^{k+1} f_{\omega_{0}}+(-1)^{n+k+1} a_{n+1} f_{d a_{n+2} \ldots d a_{k+1}}\right. \\
& \left.-(-1)^{k} f_{d a_{n+1} \ldots d a_{k}} a_{k+1}\right\}(\omega) .
\end{aligned}
$$

Thus,

$$
\begin{aligned}
(b f)_{d a_{n+1} \ldots d a_{k+1}=} & (-1)^{k+1} f_{\omega_{0}}+(-1)^{n+k+1} a_{n+1} f_{d a_{n+2} \ldots d a_{k+1}} \\
& -(-1)^{k} f_{d a_{n+1} \ldots d a_{k}} a_{k+1}(\omega) .
\end{aligned}
$$

So we have, after cancellations,

$$
(b \alpha+\alpha b) f=f,
$$

as required.

Now, from the definition of $\alpha$ it is clear that

$$
\|\alpha\| \leqq\|\phi\|
$$
clude

uniformly in all dimensions $\geqq n+1$. Thus we can apply Theorem 3.2 to con-

Theorem 5.2. Let $A$ be a Banach algebra such that for a fixed integer $n \geqq 0$, $H^{n+1}\left(A, M^{*}\right)=0$ for all dual Banach A-bimodules $M^{*}$. Then the natural map $H P^{*}(A) \rightarrow H E^{*}(A)$ is an isomorphism.

Remark. From the point of view of Hochschild cohomology of Banach algebras, dual bimodules are perhaps more natural. For example, it is known that the only commutative Banach algebras such that $H^{1}(A, M)=0$ for all bimodules $M$ are $\mathbb{C}^{n}$ [9], while our condition for $n=0$ is equivalent to amenability of $A$ which is already a large class. In [13] an independent proof of this special case, using virtual diagonals, can be found. 


\section{References}

1. Brown, R.: The twisted Eilenberg-Zilber theorem. Celebrazioni Archimedee del secolo XX, simposio di Topologia (1967), pp. 33-37

2. Connes, A.: On the cohomology of operator algebras. J. Funct. Anal. 28, 248-253 (1978)

3. Connes, A.: Non-commutative differential geometry. Pub. Math. IHES 62, 41-144 (1985)

4. Connes, A.: Entire cyclic cohomology of Banach algebras and characters of $\theta$-summable Fredholm modules. $K$-theory $1,519-548$ (1988)

5. Connes, A.: Non-Commutative Geometry. IHES, 1993

6. Cuntz, J., Quillen, D.: Algebra extension and nonsingularity. Heidelberg preprint

7. Haagerup, U.: All nuclear $C^{*}$-algebras are amenable. Invent. Math. 74, 305-319 (1983)

8. Jaffe, A., Lesniewski, A., Osterwalder, K.: Quantum $K$-theory I: The Chern character. Commun. Math. Phys. 118, 1-14 (1988)

9. Johnson, B.E.: Cohomology in Banach algebras. Mem. Am. Math. Soc. \#127, 1972

10. Karoubi, M.: Homologie cyclique et $K$-theorie. Asterisque 149 (1987)

11. Kassel, C.: Homologie cyclique, caracter de Chern et lemme de perturbation. J. Reine und Angew. Math. 408, 159-180 (1990)

12. Khalkhali, M.: On the entire cyclic cohomology of Banach algebras I: Morita Invariance. and II: Homotopy Invariance. Heidelberg preprints

13. Khalkhali, M., Phillips, J.: Entire cyclic cohomology of amenable Banach algebras

14. Klimek, S., Lesniewski, A.: A note on entire cyclic cohomology of a finite dimensional noncommutative space. Harvard preprint

Communicated by A. Connes 